\title{
Nasally inhaled pulsating aerosols: lung, sinus and nose deposition*
}

\author{
Winfried Möller ${ }^{1}$, Gülnaz Khadem Saba ${ }^{1}$, Karl Häussinger ${ }^{2}$, Sven Becker ${ }^{3}$, \\ Manfred Keller ${ }^{4}$, Uwe Schuschnig ${ }^{4}$ \\ 1 Institute for Lung Biology and Disease (iLBD), Helmholtz Zentrum München - German Research Center \\ for Environmental Health, Neuherberg, Germany \\ 2 Department of Pulmonary Medicine, Asklepios Fachkliniken München-Gauting, Gauting, Germany, \\ 3 Department of Otolaryngology, Head and Neck Surgery, Großhadern Medical Centre, Ludwigs Maximilians \\ University München, München, Germany \\ 4 Pari Pharma GmbH, Gräfelfing, Germany
}

SUMMARY

\section{INTRODUCTION}

Chronic rhinosinusitis (CRS) is a common chronic disease of the upper airways affecting approximately $10-15 \%$ of the US and European population ${ }^{(1)}$. The etiology of CRS is unclear but it is assumed that one or more factors, such as anatomical obstruction, bacterial or fungal colonization of the sinuses or allergies trigger a chronic inflammation of the nasal and paranasal mucosa. The high number of surgical interventions in patients suffering from CRS ${ }^{(2)}$ indicates that medical treatment by oral, systemic or topical drug administration is unsatisfactory.

Drug delivery to the sinuses via aerosols appears to be an inter- esting but also challenging alternative, since the paranasal cavities are virtually non-vented, poorly perfused, hollow organs protected by the efficient particle filtration function of the nose. In general, research on inhaled topical aerosol treatment of upper airway diseases is desirable but has been 'significantly neglected' ${ }^{(3)}$ compared to pulmonary drug investigations.

The only scientifically comprehensible approach of aerosol transport to the sinuses is via pressure differences using socalled 'vibrating,' 'sonic' or 'pulsating' aerosols. The first paper on vibrating aerosols was published in $1959^{(4)}$ and suggests that pressure fluctuations enhance the transport of aerosol 
particles and allows them to penetrate into non-vented areas. Another early publication ${ }^{(5)}$ identified flow induction to the sinuses by a pressure gradient to be the most important transport mechanism for aerosols. A more recent study compared the amounts of exhaled nitric oxide (NO) after administration of aerosolized NO synthase inhibitor with and without vibration (humming) in six healthy volunteers ${ }^{(6)}$ and demonstrated significant reduction of $\mathrm{NO}$ after administering the aerosol with pulsations. Möller et al. successfully demonstrated the ventilation of the sinuses via pressure pulsations using radioactive ${ }^{81 \mathrm{~m}} \mathrm{Kr}$ gas ${ }^{(7)}$ and sinus deposition of ${ }^{99 \mathrm{~m}} \mathrm{Tc}$-DTPA radiolabeled aerosol in healthy volunteers ${ }^{(8)}$. The PARI SINUS, PARI's first pulsating aerosol device, is based on a modified PARI LC SPRINT jet nebulizer and is currently used to investigate in clinical trials the therapeutic effect of mucolytics and antibiotics in cystic fibrosis $(\mathrm{CF})$ patients ${ }^{(9)}$. Encouraging results from a double-blind placebo controlled trial in $23 \mathrm{CF}$ patients using the PARI SINUS with Dornase alpha were published recently ${ }^{(9,10)}$. Pari's second generation pulsating aerosol device, the PARI Vibrent generates a pulsating dense aerosol with low velocity via a perforated vibrating membrane. The Vibrent is designed to improve delivery efficiency and handling of the device ${ }^{(11)}$.

Successful drug delivery to the sinuses requires small aerosol droplets with a mass median aerodynamic diameter (MMAD) of less than $5 \mu \mathrm{m}$, as larger droplets are filtered by the nose and are not able to reach the posterior regions of the nose. Additionally, only small particles are able to follow the induced airflow to the sinuses while larger droplets cannot penetrate into these cavities due to inertia. For this reason, the SINUS and Vibrent devices generate droplets with a MMAD of about $3 \mu \mathrm{m}$, while conventional nasal pump sprays produce $50 \mu \mathrm{m}$ particles. Droplets with a MMAD below $5 \mu \mathrm{m}$ are generally regarded to be respirable and are able to penetrate into the lungs. For optimal pressure transduction and avoidance of lung deposition, the pulsating aerosol should be administered when the soft palate is closed. The principle is similar to a nasal lavage via a 'neti pot' flushing the liquid into one nostril and exiting via the other nostril. However, if a patient is unable to close the soft palate during aerosol administration, lung deposition can occur and this may be associated with a decline in sinus deposition.

The objective of this study was to investigate in healthy volunteers with normal nasal anatomy the deposition efficiency of the Vibrent device using radiolabeled aerosols upon administration of the pulsating aerosols when the soft palate was closed in comparison to administration during nasal inhalation. As benchmark, inhalation of a radiolabeled aerosol via nasal pump spray was included. In all studies, retention kinetics was measured over a 24-hour period. Pulsating aerosols showed sinus deposition when applied during closed soft palate or during spontaneous breathing, while nasal sprays do not reach into the sinuses. Interestingly, nasal clearance is drastically prolonged for pulsating aerosols.

\section{MATERIALS AND METHODS}

\section{Human volunteers}

Eleven healthy, non-smoking volunteers participated in this study with mean age of $48 \pm 14$ years. The subjects had normal lung function and no history of allergic diseases. Normal nasal anatomy was confirmed prior to the study by MRT imaging of the head and fiber optic rhinoscopy. The study protocol was approved by the Ethical Committee of the Medical School of the Ludwig Maximilian University (Munich, Germany). Informed consent was obtained from each subject. ${ }^{81 \mathrm{~m}} \mathrm{Kr}$-gas ventilation was studied with and without pulsation in each subject. Radiolabeled aerosols were applied three times on different occasions, once via Vibrent (PARI Pharma, Starnberg, Germany) at closed soft palate, once with Vibrent during nasal breathing and one application was by a nasal spray pump (Kr-gas data not shown).

\section{Pulsating aerosol delivery system}

A pulsating aerosol was produced using the PARI Vibrent nebulizer prototype (PARI Pharma $\mathrm{GmbH}$ ) generating a fine aerosol mist of an aqueous liquid via a perforated vibrating membrane (Pari eFlow). The basic aerosol generation principle is derived from the eFlow electronic nebulizer, which is approved for pulmonary drug administration, such as Colistin (12). In the Vibrent, a pressure wave of $25 \mathrm{~Hz}$ frequency was superimposed to a low velocity aerosol stream. The mass median aerodynamic diameter (MMAD) of the aerosol generated by the Vibrent nebulizer was $3.0 \mu \mathrm{m}$ with a geometric standard deviation of 1.6. The droplet size distribution of the nebulizer was measured using the Laser Diffraction Technique and there was no significant difference in droplet size between saline and the ${ }^{99 \mathrm{~m}}$ Tc-DTPA solutions used in our study. The rate of mass output was $0.3 \mathrm{ml} / \mathrm{min}$. For aerosol delivery to the nose, the nebulizer nosepiece was inserted into the right nostril and the left nostril was connected via a flow resistor to output filter to collect the expelled aerosol. Subjects were instructed to either close their soft palate during administration of the aerosol into one nostril or to breathe in and out through the other nostril, while the mouth was closed.

\section{Nasal pump sprays}

The nasal pump spray aerosol was generated using a standard $100 \mu 1$ nasal spray pump from a major manufacturer, producing an aerosol spray of a mean droplet diameter (MMAD) of about $50 \mu \mathrm{m}$. Four milliliters of DTPA solution containing about $100 \mathrm{MBq}$ of ${ }^{99 \mathrm{~m}} \mathrm{Tc}$-activity was added to the bottle. One $100 \mu \mathrm{l}$ puff was delivered into the right and left nostril, respectively.

\section{Aerosol administration studies}

The aerosol was generated using a solution composed of ${ }^{99 \mathrm{~m}} \mathrm{Tc}-$ DTPA (Pentacis, Schering, Germany). The volunteers did not receive any treatment before application, for example decongesting nasal spray, though the nasal cycle was not eliminated. Prior to aerosol delivery the output rate of the nebulizer was measured by collecting all particles on a PALL BB50 filter. 
From the nebulizer output rate and the activity deposited on the output filter, the total nasal deposition rate was assessed.

For nasal aerosol delivery at closed soft palate, the nosepiece of the nebulizer was inserted into the right nostril, while a filter including a flow resistor was connected to the left nostril (PALL BB50 filter, Pall Corporation, New York, NY, USA). The aerosol was delivered for 20 seconds, while the subject closed the soft palate. Nebulizer and output filter were then interchanged between left and right nostril and the aerosol was delivered for an additional 20 seconds.

For aerosol administration during nasal breathing, the device was inserted into one nostril, while the other nostril was connected to a low resistance PARI filter pad (PARI GmbH) to collect the exhaled aerosol. The volunteer closed his mouth and inhaled and exhaled through the filter during aerosol application of 20 seconds. Then, the device and exhalation filter were exchanged and the 20 second application was repeated.

Nasal and lung deposition, retention and clearance were measured using planar gamma camera imaging (SCINTRON, MiE $\mathrm{GmbH}$, Seth, Germany) in combination with a low energy collimator. Anterior and lateral gamma camera images were recorded directly after inhalation as well as $0.5 \mathrm{~h}, 1 \mathrm{~h}, 2 \mathrm{~h}, 4$ $\mathrm{h}, 6 \mathrm{~h}$ and $24 \mathrm{~h}$ post inhalation. The subjects sat in front of the gamma camera head, which was positioned in the upright format, allowing a simultaneous imaging of the nose and the upper lung. Anterior and lateral images were recorded with and without a nasal lead shield (Figure 1). Masking the centrally deposited activity allowed clear visualization of the deposited activity in the maxillary and ethmoid sinuses without detecting activity from the central nose. Count rates in selected regions of interest (ROI's) were analyzed using the ImageJ software package. ROI's generated from previous ${ }^{81 \mathrm{~m}} \mathrm{Kr}$ ventilation images were used and included the nasal airways and the sinuses ${ }^{(7)}$. Count rates were corrected for background activity and for radioactive decay. The results were not corrected for gamma ray attenuation, which may lead to an underestimation of sinonasal deposition rates. Data were evaluated with Statgraphics Centurion XV software, using a significance level of $\mathrm{p}<0.05$.

\section{RESULTS}

Aerosol deposition with nasal spray

$100 \%$ of the radioactivity delivered by the pump spray deposited in the nose and no deposition was observed in the lungs. The deposition in the maxillary sinuses was $<1 \%$ and the small fraction detected in the sinus ROIs was probably caused by gamma ray scattering. In the lateral gamma camera images, no activity was found in the ethmoid and sphenoid sinuses in most of the volunteers (Figure 2).

\section{Pulsating aerosol deposition when the soft palate was closed} The first image recorded immediately after aerosol delivery did not show any aerosol deposition in the chest or abdomen
Table 1. Nasal clearance of the administered activity after different modes of application.

\begin{tabular}{lcc}
\hline Administration route & $\begin{array}{l}\text { Time to 50\% clear- } \\
\text { ance from nose, } \\
\text { hours }\end{array}$ & $\begin{array}{l}\text { Time to 75\% clear- } \\
\text { ance from nose, } \\
\text { hours }\end{array}$ \\
\hline $\begin{array}{l}\text { Nasal pump spray } \\
(100 \mu \text { l per nostril) }\end{array}$ & $0.41 \pm 0.48^{* *}$ & $1.24 \pm 1.31^{* *}$ \\
$\begin{array}{l}\text { Pulsating aerosol at } \\
\text { closed soft palate }\end{array}$ & $0.92 \pm 0.59$ & $4.40 \pm 3.36$ \\
$\begin{array}{l}\text { Pulsating aerosol } \\
\text { during nasal breathing }\end{array}$ & $1.00 \pm 0.56$ & $5.48 \pm 2.64$ \\
\hline
\end{tabular}

Data represent mean \pm standard deviation, ${ }^{* *}: \mathrm{p}<0.01$ compared to vibrating aerosol administration with closed soft palate.

(stomach) region confirming the tight closure of the soft palate during aerosol delivery. The anterior image with and without nasal shield demonstrated clear deposition of radioactivity in the maxillary sinuses in all volunteers. The lateral view showed deposition in the nasal roof, the sphenoid and ethmoid sinuses, whereas no significant deposition was seen in the frontal sinuses (Figure 3). $69.7 \pm 15.6 \%$ of the radioactivity delivered as pulsating aerosol deposited in the nose, while $30.3 \pm 15.7 \%$ were found on the exit filter. The mean activity in the ethmoid and maxillary sinuses was $7.0 \pm 1.4 \%$ of the nasally deposited activity.

\section{Pulsating aerosol deposition during nasal breathing application} When the pulsating aerosol was applied during nasal breathing, $10.0 \pm 4.2 \%$ of the emitted radioactivity was detected in the lungs (Figure 4), $62.6 \pm 12.7 \%$ in the nose including the sinuses and $26.8 \pm 12.7 \%$ were exhaled (collected on exit filter). $4.5 \pm 1.1$ of the nasally deposited activity was found in the sinuses. Again, deposition in the maxillary, ethmoid and sphenoid sinuses could be demonstrated (Figure 5).

\section{DTPA clearance from the nasal cavity}

The nasal ${ }^{99 \mathrm{~m}}$ Tc-DTPA retention until $50 \%$ and $75 \%$ clearance was reached is shown in Table 1 and Figure 6. Clearance from the nasal cavity is significantly prolonged when pulsating aerosols were administered compared to nasal pump spray $(\mathrm{p}<0.05)$. No significant difference regarding clearance was found when pulsating aerosol administrations were conducted with closed soft palate or during nasal breathing ( $p>0.36$ ). Figure 4 also shows high activity accumulation in the stomach confirming mucociliary clearance from the nasal cavity into the throat followed by swallowing of the activity.

\section{DISCUSSION}

As expected, nasal pump sprays did not deliver significant amounts of aerosol into the posterior part of the nose including sinuses, as the coarse aerosol of $50 \mu \mathrm{m}$ diameter droplets deposits predominantly at the nasal valve and inferior turbinate as already described previously ${ }^{(12,13)}$. 


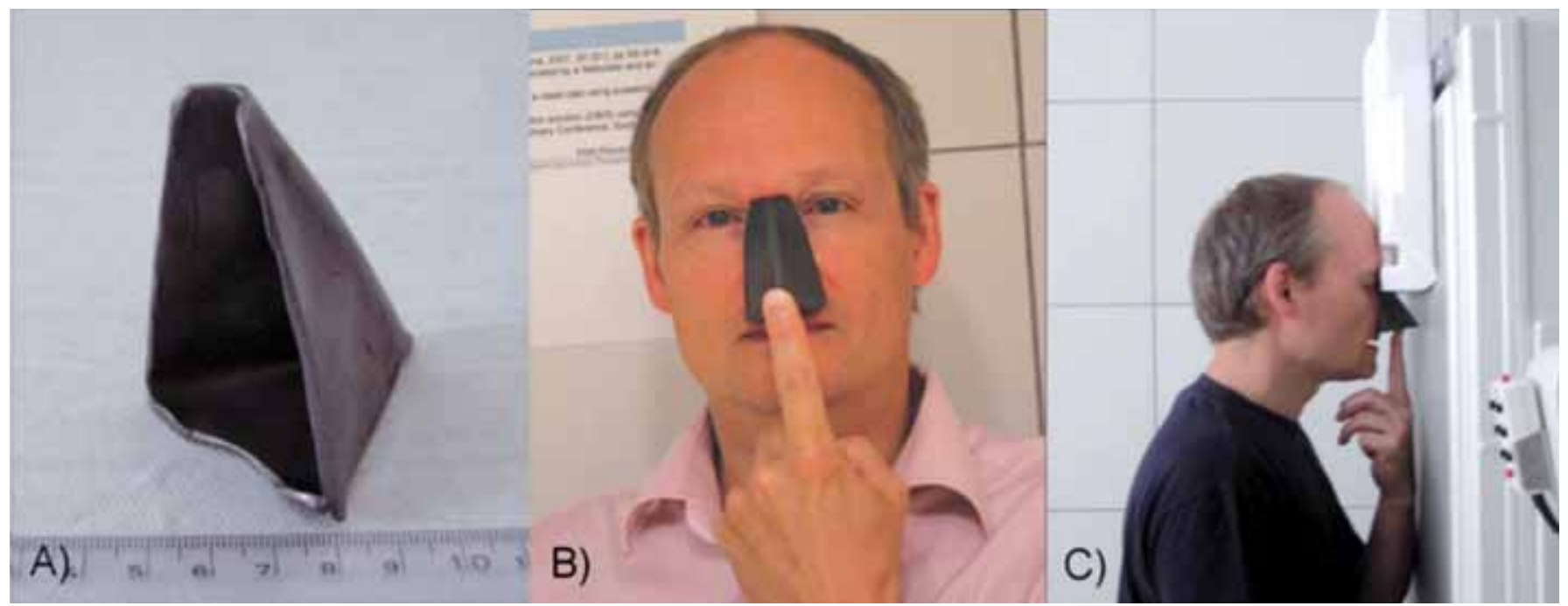

Figure 1. Nasal lead shield (A), volunteer with nasal lead shield (B), and volunteer during anterior gamma camera imaging with nasal shield (C).

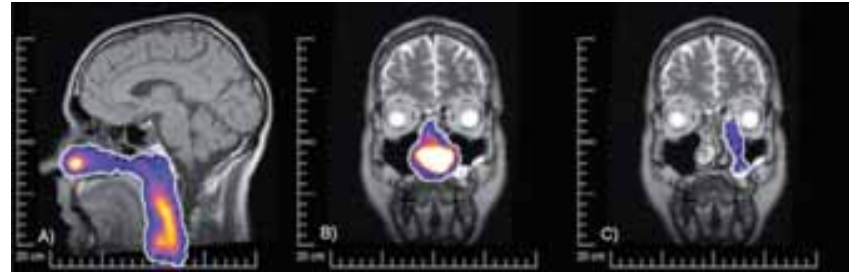

Figure 2. Gamma camera image of ${ }^{99 m}$ Tc-DTPA activity distribution of nasal pump spray, superimposed to MRI image in lateral (A) and anterior view without a nasal shield (B) and with a nasal shield (C).

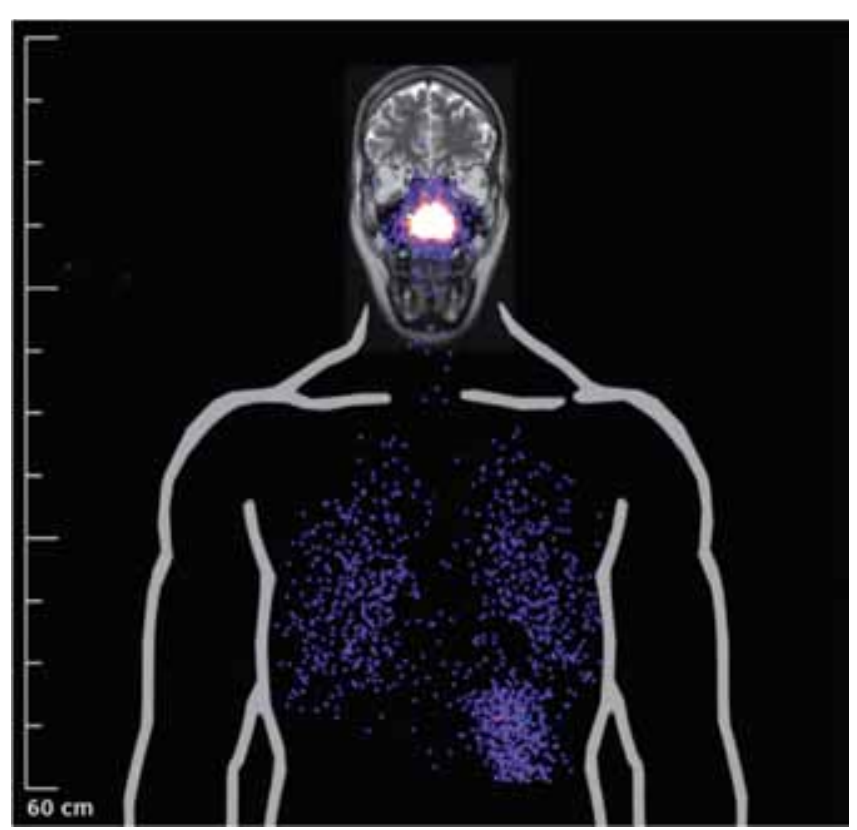

Figure 4. Gamma camera image of ${ }^{99 \mathrm{~m} T c-D T P A}$ activity distribution of pulsating aerosol administration during nasal breathing, showing activity in the nose, lungs and stomach.

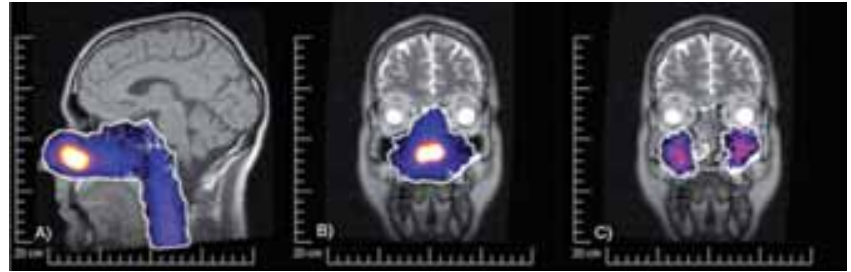

Figure 3. Gamma camera image of ${ }^{99} \mathrm{~m} \mathrm{Tc}-\mathrm{DTPA}$ activity distribution of pulsating aerosol at closed soft palate aerosol administration in lateral (left) and anterior view without a nasal shield (middle) and with a nasal shield (right).

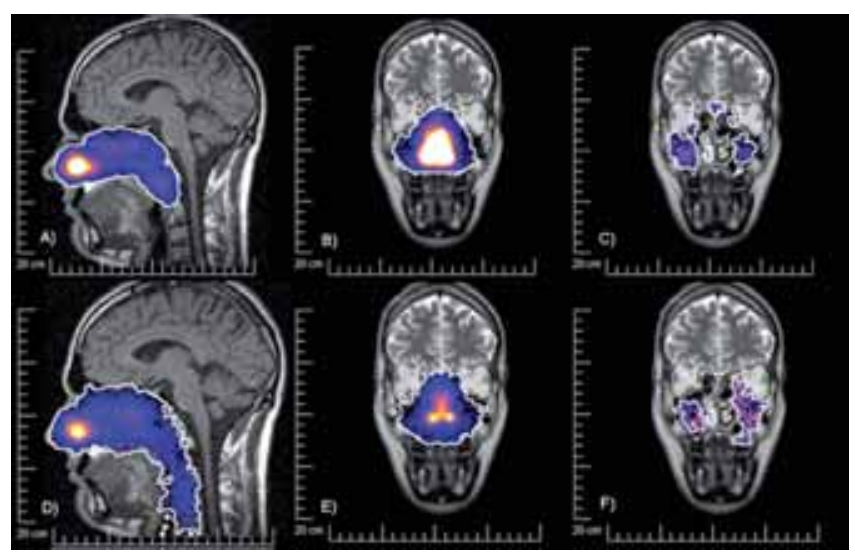

Figure 5. Comparison of different administration routes of the pulsating aerosol: upper series (A-C) during nasal breathing; lower series (D-F) at closed soft palate. Graphs show lateral images (A, D) and anterior images without lead shield (B, E) and with nasal shield (C, F). 


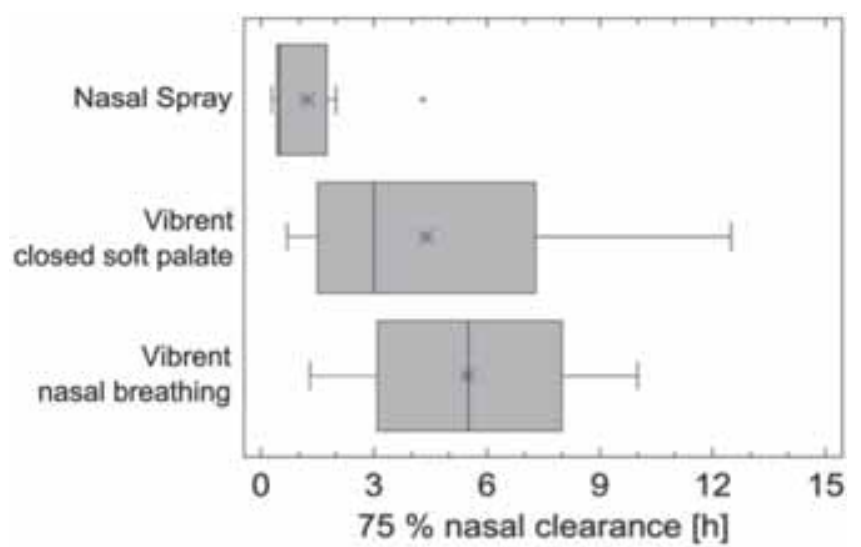

Figure 6. Box plot of duration of $75 \%$ clearance of the administered ${ }^{99 \mathrm{~m}}$ Tc-DTPA after using nasal pump sprays and during pulsating aerosol delivery at closed soft palate and during nasal breathing. Star denotes mean values.

\section{Aerosol deposition distribution}

Using the masking technique with a nasal lead shield, deposition of pulsating aerosols in the maxillary sinuses, as well as in the ethmoid and sphenoid sinuses could be clearly verified. While qualitative proof of (maxillary) sinus deposition is straight forward, the quantitative determination is not as evident. The deposited activity is attenuated by the surrounding bone and tissue making recovery calculations impossible. The overall attenuation factor in these experiments was around 1.5 to 2 , whereas the attenuation of more posteriorly deposited activity is most likely higher than for activity in the central and anterior part of the nose. Attenuation factors for different regions of the nose could be calculated upon the Hounsfield numbers of CT scans, but these were not available for the healthy volunteers. Hence, we decided not to correct the gamma camera counts for the attenuation factors at all, leading possibly to an underestimation of the peripherally deposited activity and an overestimation of the activity in the anterior nasal regions. However, total nasal aerosol deposition was evaluated based on output and filter measurements, which do not depend on attenuation, and therefore represent reliable values. Nevertheless, the methodology of evaluating activity counts in different ROIs defined upon ventilation experiments as described by Möller ${ }^{(7,8)}$ is reproducible, objective and allows the comparison of devices and application routes.

\section{Influence of nasal breathing on nasal and lung deposition}

According to these investigations a closed soft palate is favorable for the delivery of aerosolized drugs to the paranasal cavities while inserting the nose piece of the Vibrent in one nostril and allowing the aerosol to expell via the other one, which is in addition equipped with a flow resistor. This route offers two major advantages: it prevents lung deposition and the additional flow resistor enhances the pressure pulsations in the sinuses enhancing sinus ventilation. The device delivers an aerosol at pulsating flow rates, which produce pressure fluctuations in the presence of a flow resistor. It should be kept in mind, that the nose itself, including the nasal valve, exhibits flow resistances, but adding an additional (external) restriction ascertains an enhanced pressure drop in the nose including enhanced gas exchange with the sinuses. However, if subjects are unable to close their soft plate, the flow resistance of the nose might still apply. Especially during exhalation through the nose a 'natural' pressure drop is generated, which may enable aerosol transport to the sinuses. This hypothesis could be confirmed in our experiments, since we found $7 \%$ sinus deposition when the soft palate was closed and $4.5 \%$ during nasal breathing $(\mathrm{p}<0.01)$. These findings indicate that a higher pressure drop results in higher sinus deposition and the nasal flow resistance is enabling increased sinus drug delivery. On the other hand, nasal inhalation yields a lung deposition of about $10 \%$ of the emitted radioactivity, compared to $33 \%$ to $58 \%$ lung deposition after nasal inhalation of conventional, non pulsating aerosols ${ }^{(13)}$. Nevertheless, lung deposition must be considered particularly with respect to antibiotics and potential resistance aspects.

\section{Radiotracer clearance from the nose}

Our clearance data for the nasal pump sprays are in good agreement with previously published studies ${ }^{(13-16)}$. The dominant clearance mechanism from the nose is mucociliary clearance into the throat, which is then swallowed, resulting in activity accumulation in the stomach, which is visible from Figure 4 . Suman ${ }^{(13)}$ reported clearance of $25 \%$ - $40 \%$ of nasally administered DTPA aerosols within 30 minutes when delivered from a nasal pump spray or nasal nebulizer, respectively. Surprisingly, clearance of the radiotracer from the nose is about 5-fold slower for pulsating aerosols compared to nasal pump sprays. So far, we don't have a convincing explanation for the slower clearance of pulsating aerosols, but this finding is reproducible and was also observed for the PARI SINUS ${ }^{(7)}$. In summary, this retarded clearance kinetics of pulsating aerosols was now observed in over 20 subjects warranting further experiments to better understand this mechanism.

However, the data of this study were obtained in healthy volunteers with normal nasal anatomy and the results may not directly be applicable to patients with CRS, having most often obstructed or clogged nasal pathways or ostia, which may prevent gas and aerosol penetration to the sinuses. Currently, we are running a clinical study to investigate pulsating aerosol deposition in CRS patients, pre and post sinus surgery.

\section{ACKNOWLEDGEMENT}

The study was supported by a research grant from Pari GmbH, Starnberg, Germany, and from the German Ministry for Education and Research (BMBF, 03FPE00028).

\section{AUTHOR CONTRIBUTIONS}

US, KH, MK and WM designed the study and prepared and reviewed the manuscript. WM, US, GKS and GM conducted the study and analyzed the data. 


\section{DISCLOSURES}

WM received a research grant from PARI GmbH in 2009 for testing nasal drug delivery devices. All other authors declare no conflicts of interests.

\section{REFERENCES}

1. Pleis JR, Lucas JW. Summary health statistics for U.S. adults: National Health Interview Survey, 2007. Vital and health statistics Series 10, Data from the National Health Survey. 2009; 1-159.

2. Schappert SM, Rechtsteiner EA. Ambulatory medical care utilization estimates for 2006. National health statistics reports. 2008; $1-29$.

3. Laube BL. Devices for aerosol delivery to treat sinusitis. J Aerosol Med. 2007; 20: S5-S18.

4. Guillerm R, Badre R, Flottes L, et al. Nouveau procede assurant la penetration des aerosols dans les sinus. (A new method of aerosol penetration into the sinuses). Presse Med. 1959; 67: 1097-1098.

5. Kauf H. Eindringvermögen von Aerosolen in Nebenräume (Penetration ability of aerosols into secondary spaces). Eur Arch Otorhinolaryngol. 1968; 190: 95-108.

6. Maniscalco M, Sofia M, Weitzberg E, et al. Sounding airflow enhances aerosol delivery into the paranasal sinuses. Eur J Clin Invest. 2006; 36: 509-513.

7. Möller W, Schuschnig U, Meyer G, et al. Ventilation and aerosolized drug delivery to the paranasal sinuses using pulsating airflow - a preliminary study. Rhinology. 2009; 47: 405-412.

8. Möller W, Schuschnig U, Khadem Saba G, et al. Pulsating aerosols for drug delivery to the sinuses in healthy volunteers. Otolaryngol Head Neck Surg. 2010; 142: 382-388.

9. Mainz J, et al. Sinonasal inhalation of dornase alfa reduces rhinosinusitis symptoms in CF. Results of a DBPC-cross-over study. J Cyst Fibrosis 2010; 9, Suppl 1, S23.

10. Mainz JG, Schiller I, Ritschel C, et al. Sinonasal inhalation of dornase alfa in CF: A double-blind placebo-controlled cross-over pilot trial. Auris Nasus Larynx. 2011; 38: 220-227.
11. Schuschnig U, Keller M, Klopfer E, et al. Drug Delivery to the Nasal and Paranasal Cavities - Critical Cast Dimensions and Aerosol Dynamics. In: Respiratory Drug Delivery 2008 Arizona. Dalby RN, Byron PR, Peart J, Suman JD, Farr SJ, Young PM, editors. Scottsdale, Arizona, USA: Virginia Commonwealth University; 2008. p. 227-38.

12. Knoch M, Keller M. The customised electronic nebuliser: a new category of liquid aerosol drug delivery systems. Expert Opin Drug Deliv. 2005; 2: 377-390.

13. Suman JD, Laube BL, Dalby R. Comparison of nasal deposition and clearance of aerosol generated by nebulizer and an aqueous spray pump. Pharm Res. 1999; 16: 1648-1652.

14. Kimbell JS, Segal RA, Asgharian B, et al. Characterization of Deposition from Nasal Spray Devices Using A Computational Fluid Dynamics Model of The Human Nasal Passages. J Aerosol Med. 2007; 20: 59-74.

15. Newman SP, Moren F, Clarke SW. Deposition pattern of nasal sprays in man. Rhinology. 1988; 26: 112-120.

16. Bryant ML, Brown P, Gurevich N, et al. Comparison of the clearance of radiolabelled nose drops and nasal spray as mucosally delivered vaccine. Nucl Med Commun. 1999; 20: 171-174.

\section{Dr. Winfried Möller}

Helmholtz Zentrum München -

German Research Center for Environmental Health

Institute for Lung Biology and Disease (iLBD)

Ingolstädter Landstraße 1

85764 Neuherberg

Germany

Tel: + 49-89-3187 1881

Fax: + 49-89-3187 19188

E-mail: moeller@helmholtz-muenchen.de 